\title{
Video Article \\ An All-in-one Sample Holder for Macromolecular X-ray Crystallography with Minimal Background Scattering
}

\author{
Christian G. Feiler ${ }^{1}$, Dirk Wallacher ${ }^{2}$, Manfred S. Weiss ${ }^{1}$ \\ ${ }^{1}$ Macromolecular Crystallography (HZB-MX), Helmholtz-Zentrum Berlin \\ ${ }^{2}$ Department Sample Environment, Helmholtz-Zentrum Berlin
}

Correspondence to: Manfred S. Weiss at msweiss@helmholtz-berlin.de

URL: https://www.jove.com/video/59722

DOI: doi:10.3791/59722

Keywords: Biochemistry, Issue 149, Sample holder, in situ data collection, cryo-protection, ligand-soaking, crystallization platform, hanging-drop, automation, SPINE standard, and macromolecular crystallography

Date Published: $7 / 6 / 2019$

Citation: Feiler, C.G., Wallacher, D., Weiss, M.S. An All-in-one Sample Holder for Macromolecular X-ray Crystallography with Minimal Background Scattering. J. Vis. Exp. (149), e59722, doi:10.3791/59722 (2019).

\section{Abstract}

Macromolecular X-ray crystallography (MX) is the most prominent method to obtain high-resolution three-dimensional knowledge of biological macromolecules. A prerequisite for the method is that highly ordered crystalline specimen need to be grown from the macromolecule to be studied, which then need to be prepared for the diffraction experiment. This preparation procedure typically involves removal of the crystal from the solution, in which it was grown, soaking of the crystal in ligand solution or cryo-protectant solution and then immobilizing the crystal on a mount suitable for the experiment. A serious problem for this procedure is that macromolecular crystals are often mechanically unstable and rather fragile. Consequently, the handling of such fragile crystals can easily become a bottleneck in a structure determination attempt. Any mechanical force applied to such delicate crystals may disturb the regular packing of the molecules and may lead to a loss of diffraction power of the crystals. Here, we present a novel all-in-one sample holder, which has been developed in order to minimize the handling steps of crystals and hence to maximize the success rate of the structure determination experiment. The sample holder supports the setup of crystal drops by replacing the commonly used microscope cover slips. Further, it allows in-place crystal manipulation such as ligand soaking, cryo-protection and complex formation without any opening of the crystallization cavity and without crystal handling. Finally, the sample holder has been designed in order to enable the collection of in situ X-ray diffraction data at both, ambient and cryogenic temperature. By using this sample holder, the chances to damage the crystal on its way from crystallization to diffraction data collection are considerably reduced since direct crystal handling is no longer required.

\section{Video Link}

The video component of this article can be found at https://www.jove.com/video/59722/

\section{Introduction}

The knowledge of the three-dimensional structure of biological macromolecules constitutes an important cornerstone in all basic biological, biochemical and biomedical research. This even extends to certain translational aspects of such research, such as for instance drug discovery. Among all methods for obtaining such three-dimensional information at atomic resolution X-ray crystallography is the most powerful and the most prominent one as is evidenced by the fact that $90 \%$ of all available structural information is contributed by X-ray crystallography ${ }^{1}$. The major prerequisite of X-ray crystallography, which is at the same time its major limitation, is that diffraction-quality crystals have to be produced and prepared for the diffraction experiment. This step still constitutes one of the major bottlenecks of the method.

Historically, diffraction data from protein crystals were collected at ambient temperature. Individual crystals were carefully transferred into glass or quartz capillaries prior to data collection, mother liquor was added to the capillaries so that the crystals would not dry out and the capillaries were sealed $^{2,3,4}$. Since the 1980 s, it became more and more apparent that due to the ionizing properties of X-radiation and the imminent radiation sensitivity of macromolecular crystals, data collection at ambient temperature poses severe limitations on the method. Consequently, approaches were developed to mitigate radiation damage effects by cooling macromolecular crystals down to $100 \mathrm{~K}$ and to collect diffraction data at such low temperature ${ }^{5,6}$. For working at low temperatures, the mounting of the samples in capillaries became impractical due to the low rate of heat transfer. In spite of this, there are ongoing efforts to also use capillaries, in particular from counter-diffusion crystallization experiments, for low-temperature diffraction work ${ }^{7,8}$, but, irrespective of that, it became the standard approach in macromolecular crystallography to mount macromolecular crystals held by a thin film of mother liquor inside a thin wired loop ${ }^{9,10}$. Even though a number of improvements (e.g., the introduction of lithographic loops and similar structures ${ }^{11}$ ) have been made over time to this loop-based mounting, the basic principles that were developed in the early 1990s are still in use today. It may be safely stated that most diffraction data collections on macromolecular crystals nowadays still rely on this approach ${ }^{5}$.

Over time, there were some interesting new developments and modifications of the loop-based mounting method, but these approaches have so far not been widely adopted in the community. One is the so-called loop-less mounting of crystals, which was developed to achieve lower 
background scattering ${ }^{12,13,14}$. Another one is the use of graphene sheaths to wrap the crystalline samples and to protect them from drying out. Graphene is a well-suited material in that respect because of its very low X-ray scattering background ${ }^{15}$.

More recently, developments in the field of sample mounts were mainly focused on standardizing the mounts with the aim of increasing sample throughput ${ }^{16}$ or on designing mounts, which can hold more than one sample ${ }^{17}$, such as for instance patterned membranes on a silicon frame, which are capable of holding hundreds of small crystals mostly in the field of serial crystallography ${ }^{18,19,20,21,22}$.

All of the sample mounting methods discussed so far still require some degree of manual intervention, which means that there is an inherent danger of causing mechanical damage to the sample. Therefore, novel approaches are being sought by engineering the sample environment such that diffraction data of crystals can be collected within their growth environment. One such method is termed in situ or plate-screening ${ }^{23,24}$ and it is already implemented at a number of macromolecular crystallography beamlines at various synchrotron sources worldwide ${ }^{25}$. However, the use of this method is limited by the geometrical parameters of the crystal plate and the space available around the sample point of the instrument.

Yet another approach is realized in the so-called CrystalDirect system ${ }^{26}$. Here, entire crystallization drops are harvested automatically. The foils on which the crystals have been grown are custom-cut using a laser and directly used as the sample holder ${ }^{27}$.

In the work described here, the aim was to develop a sample holder, which would allow a user to move the crystalline sample from its growth chamber to the data collection device without touching it and which would enable the user to manipulate the sample easily. Since many researchers in the field of macromolecular crystallography are still using the 24-well crystallization format for optimizing crystal growth by modifying conditions identified in large screening campaigns, the new sample holder was designed to be compatible with this format. In the following, the design of the new sample holder will be described and the handling and the performance of the sample holder for in situ data collection and ligand soaking will be demonstrated. Finally, the suitability of this new sample holder as well as its limitations for the various work steps will be discussed.

\section{Protocol}

CAUTION: For all subsequent work, it is very important that the yellow-colored polyimide foil must not be touched with unprotected fingers, because of possible contaminations to the sample holder. Also, the usage of protected forceps is highly recommended.

\section{The sample holder}

1. Use one of the three types of sample holder. NOTE: Three different versions of the new developed sample holder are shown in Figure 1. All of them contain a black plastic support structure, an airtight $\mathrm{COC}$ foil on the outside and a microporous structured polyimide foil on the inside. Type 1 (Figure 1A) contains a fixed outer plastic ring, whereas for types 2 and 3 (Figure 1B,1C) the outer ring can be broken off mechanically at the designated respective break points for use in automated sample transfer systems (see red arrows in Figure 1B). The design of the sample holders allows the setup of multiple crystallization drops on the yellow polyimide foil. It does not compromise the monitoring of the crystallization experiment, as the material is highly transparent for visible light. The $21 \mu \mathrm{m}$ thick polyimide foil also features $5 \mu \mathrm{m}$ pores, which allows simple crystal manipulation by soaking later on. Since the transmission of X-rays is close to 1.0 at all commonly used diffraction data collection energies in macromolecular crystallography, the contribution of the foil to the background scattering in a diffraction experiment is negligible ${ }^{28}$.

\section{Setting up crystallization drops}

1. Create a clean and dust-free surface using a damp lint-free cloth. Take one sample holder from its box and gently place it, yellow foil facing up, on the cleaned surface to avoid damage or unwanted puncture of the backside COC foil.

2. Set up the crystallization drops with a maximum recommended volume of $2 \mu \mathrm{L}$ on the yellow foil as it would be done on commonly used cover slides. Place the drops gently to avoid any rupture or piercing of the foil using a pipette. On a sample holder of type 1 (Figure 2A) up to three drops can be placed, whereas on sample holders of type 2 and 3 two drops are the recommended maximum (Figure 2C).

3. Flip the sample holder over and place it onto a pre-greased cavity of a 24 -well Linbro style plate. Use the positioning aids (see red arrows in Figure 1A) of the sample holder to guide it to its optimal position.

4. Ensure the correct position of the sample holder in order to avoid unwanted evaporation (Figure 2A).

\section{Observing crystal growth}

1. By placing the crystallization plate under a transmission light microscope, with or without polarizers, monitor crystal growth without any disturbance of the experiment (Figure 4).

2. When using the smaller 18-mm sample holders of type 3 (Figure 1C), which were designed for use on SBS footprint plates, use an imaging robot capable of handling SBS-footprint plates to monitor the crystal growth in a more automated way.

\section{Crystal manipulation}

NOTE: It is recommended to perform all subsequent steps under a transmission light microscope.

\section{Cryo-protection}

1. Gently pierce the outer $\mathrm{COC}$ foil using a fine cannula. Make sure the inner yellow foil remains untouched. The puncture should be right next to the drop that is to be manipulated (Figure $3 \mathrm{~A}, 3 \mathrm{C}$ ). 
2. Use a fine paper wick and insert it in the poked hole. Carefully push the wick forward until it touches the yellow polyimide foil. Keep the wick in contact with the perforated foil. The wick will suck away all excess solution. The time required for complete liquid removal depends on the viscosity of the solutions and the mother liquor composition (Figure 3B).

3. After all liquid is sucked away, gently retract the paper wick. Remember the position of the drop, since it may not be visible after removal of the mother liquor.

4. Take a standard pipette to apply a small volume of cryo-protectant solution, max. $3 \mu \mathrm{L}$, using an extruded tip (e.g., a gel loading tip) through the same hole. Once the liquid is dispensed, retract the tip. The porosity of the yellow foil allows for diffusion across the foil. The time to attain cryo-protection of your crystals highly depends on the employed solution and its components.

5. To reseal the self-healing $\mathrm{COC}$ foil, gently place a protected finger on the hole for about $1 \mathrm{~s}$ and slide it across the puncture. The slight pressure in combination with the elevated temperature will promote the resealing of punctures, which are not too large.

\section{Ligand soaking}

NOTE: Excess mother liquor may be removed before ligand soaking. To do so, follow the steps described in 4.1.1 to 4.1.3.

1. Dissolve the ligand in mother liquor in the desired concentration in a reaction tube.

2. Spin the solution for 10 minutes at $12,000 \times g$ in order to remove insoluble particles. Use a temperature-controlled centrifuge if needed.

3. Gently place a volume of max. $3 \mu \mathrm{L}$ of ligand containing solution in the gap between the $\mathrm{COC}$ foil and the polyimide film using a long, extruded pipet tip. Retract the tip.

4. To reseal the self-healing $\mathrm{COC}$ foil, gently place a protected finger on the hole for about $1 \mathrm{~s}$ and slide it across the puncture (see also 4.1.5).

5. Incubate the experiment for some time in order to allow for diffusion across the membrane. The soaking time highly depends on the viscosity of the diffusing solution and its components ${ }^{29}$.

6. Repeat steps 4.2 .1 to 4.2 .5 multiple times to subsequently soak different ligands.

\section{In situ diffraction data collection at ambient temperature}

NOTE: In order to minimize solvent scattering, remove excess solution before data collection.

1. Ensure a stable humidity controlled beamline environment with pre-established conditions ${ }^{30}$.

2. Gently lift the transparent $\mathrm{COC}$ foil at the designated point using forceps and peel it off like one would remove the lid from a yogurt cup ( Figure 6B).

3. Gently lift the sample holder from its cavity and insert it immediately into a pre-prepared magnetic sample holder base. No glue is necessary for this step (Figure 6B).

4. Apply gentle pressure to ensure the correct positioning of the sample holder within the base.

5. Mount the sample holder on a beamline goniometer and ensure correct positioning of the holder. Depending on goniometer geometry the sample holder can be rotated by up to $160^{\circ}$ without causing any shadowing during the diffraction experiment.

6. Use a paper wick and gently touch the yellow polyimide foil from the backside to remove excess mother liquor. Please note, that at that stage ligand soaking or cryo-protection may be performed just as well. The sample is now ready for centering and diffraction data collection.

7. When using a sample holder with removable outer ring, apply gentle pressure by holding on to the outer ring and break it off at the designated break points (Figure 6C). The sample is now ready for centering and diffraction data collection.

\section{In situ diffraction data collection at cryogenic temperature}

NOTE: It is recommended to remove residual mother liquor from the sample by performing the steps 4.1.1. to 4.1.3. before continuing with the next steps to minimize solvent scattering. Most samples may be transferred to liquid nitrogen without prior cryo-protection ${ }^{31}$. If cryo-protection is needed, see steps 4.1.1. to 4.1.5.

1. Gently lift the COC foil at the designated point using a forceps and peel it off (see step 5.1.2) (Figure 6A).

2. Take the sample holder off the cavity and mount it on a magnetic sample holder base. Gentle pressure may be applied in order to ensure correct and tight fitting (see step 5.1.5, Figure 6B).

NOTE: The symmetrically arranged designated break points allow for simple removal of the outer ring of the sample holder by applying gentle pressure (see step 5.1.8., Figure 6C). Now, the sample holder is ready and can be plunged into liquid nitrogen. The geometry of the sample holder types 2 and 3 (Figure 1B,1C) allows their transfer into standard SPINE sample vials, which can be used for robot assisted sample mounting (Figure 6D).

\section{Representative Results}

The sample holder type 1 has been designed so that it fits onto a well of a 24-well Linbro style plate. Each individual sample holder contains positioning aids on either side of the outer rim in order to ensure optimal positioning on the rim of the well (Figure 1A, Figure 2A). Up to three individual crystallization drops of maximum volume $2 \mu \mathrm{L}$ each can be placed onto the yellow polyimide foil (Figure 2B). For sample holders of types 2 and 3 , it is recommended to set a maximum of two drops of maximum volume $2 \mu \mathrm{L}$ each. 24 sample holders can be fitted onto one 24 well Linbro plate (Figure 3D). 
A crystallization experiment on a 24-well Linbro plate using sample holder type 1 was set up. $1 \mu \mathrm{L}$ of hen egg-white lysozyme solution (15 mg/ $\mathrm{mL}$ ) was mixed with $1 \mu \mathrm{L}$ of mother-liquor comprising $50 \mathrm{mM} \mathrm{NaAc} \mathrm{pH} \mathrm{4.7,500} \mathrm{mM} \mathrm{NaCl}$ and 25\% (w/v) PEG-6000 on the yellow polyimide foil on the sample holder (Table 1). The drop was equilibrated at $293 \mathrm{~K}$ against $500 \mu \mathrm{L}$ of mother-liquor and crystals of the size $40-50 \mu \mathrm{m}$ were observed after 5 hours (Figure 4). Crystal growth can be observed using a transmission light microscope (Figure 4) with or without a polarizer. High transparency films ensure best observation and monitoring of crystal growing conditions using both, a conventional light microscope or an automated crystal imaging system. Crystal growth observation using UV-light was not tested.

After removing the mother liquor from around the crystals, a sample holder with hen egg-white lysozyme crystals was taken from the crystallization plate and placed in a humidity-controlled airstream on HZB-MX beamline $14.3^{32}$. Diffraction data were collected at ambient temperature in $1^{\circ}$-increments using a $150 \mu \mathrm{m}$ beam at $13.8 \mathrm{keV}$ energy with $4 \times 10^{10}$ photons $/ \mathrm{s}$ and an exposure time of $5 \mathrm{~s}$ per image. A typical diffraction image is shown in Figure 5. No elevated background scattering on the diffraction image can be detected. Further experimental details as well as associated data processing statistics are listed in Table 2.
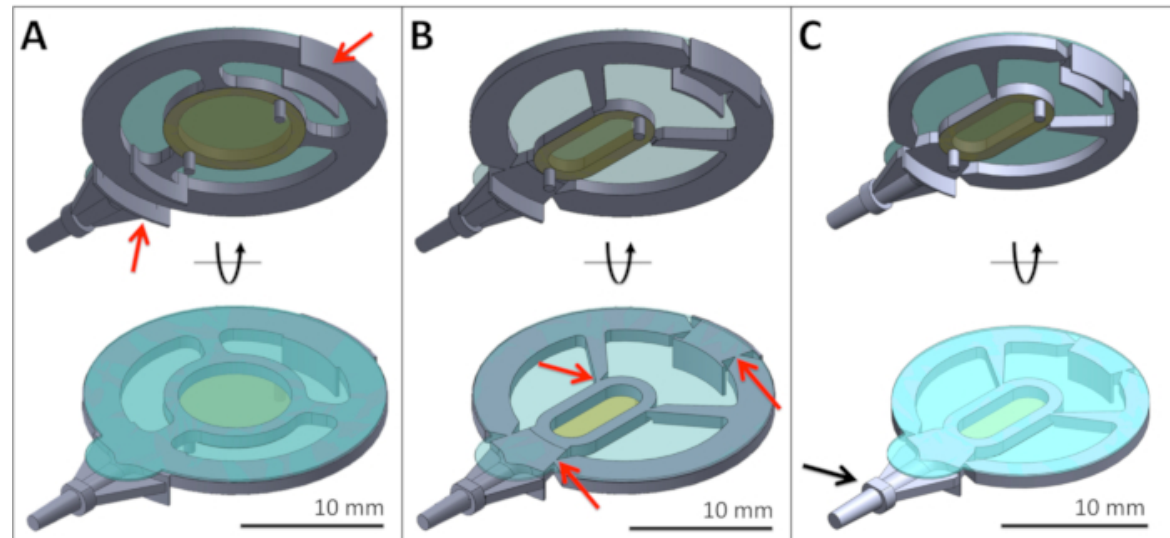

Figure 1: Schematic view of the new sample holders. The sample holders consist of a black plastic support, which is covered on the outer side with an amorphous cyclic olefin copolymer (COC) foil. This foil (colored in blue) is highly transparent and self-healing. It also ensures gas tightness of the experiment. The inner foil (colored in yellow) is made of bio-inert polyimide, which is highly transparent for X-rays. On this foil, the crystallization drops can be placed.The outer rim of the sample holder contains two positioning aids indicated by the red arrow (panel $\mathbf{A}$ ), which allows accurate placement of the sample holder on the individual pre-greased cavity of the crystallization plate. (A) Sample holder (type 1) with $22 \mathrm{~mm}$ diameter with a fixed external support ring. (B) Sample holder (type 2) with $22 \mathrm{~mm}$ diameter with removable external support ring. (C) Sample holder (type 3) with $18 \mathrm{~mm}$ diameter with removable external support ring. The latter two have been developed for using them in a high-throughput fashion with automated sample mounting robots using SPINE standard. The designated break points are highlighted by the red arrows in panel $\mathbf{B}$. The black arrow in panel $\mathbf{C}$ indicates the positioning marker. The protruding pins at the outer perimeter of the yellow foil are necessary to align the polyimide foil during the production process. Please click here to view a larger version of this figure.
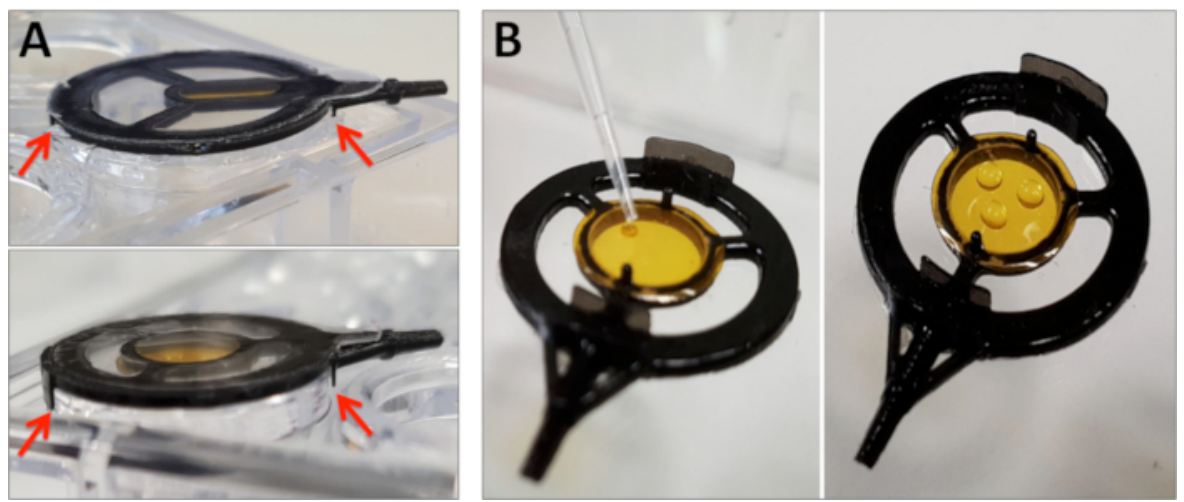

Figure 2: The sample holder may be used on a 24-well Linbro plate in the same way as the commonly used microscope cover slips. It seals the cavity airtight. Positioning aids ensure the correct positioning of the sample holder on the cavity (red arrows in panel A). Up to three individual drops may be placed onto a type 1 sample holder (panel B), whereas the recommended maximum number of drops placed on a type 2 or 3 sample holder is two. The maximum recommended volume for each drop is $2 \mu \mathrm{L}$. Please click here to view a larger version of this figure. 

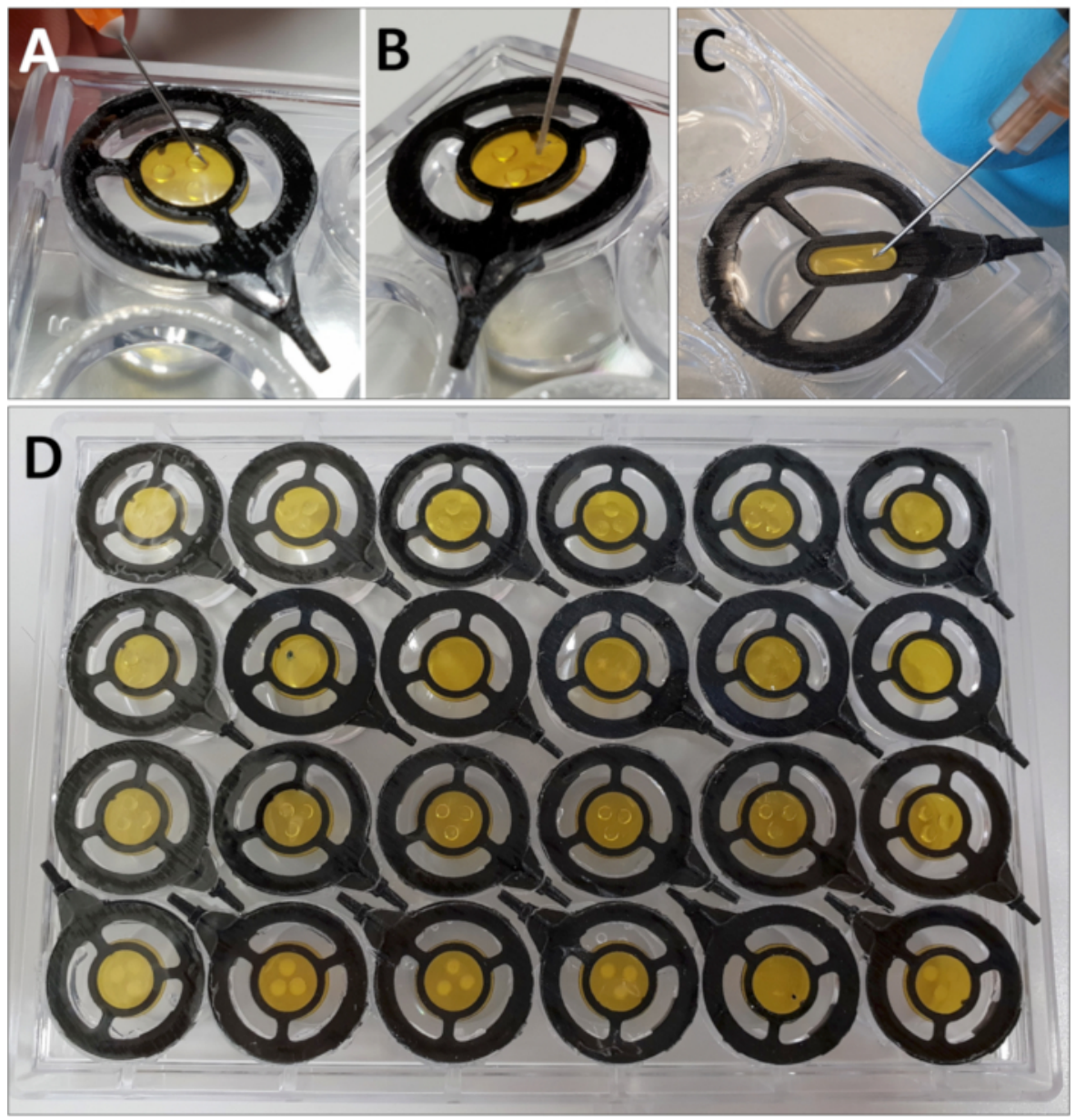

Figure 3: 24 type 1 sample holders fit on a 24-well plate. The sample holders can be placed in two orientations on the 24-well plate as indicated (panel D). A cannula is used to pierce the back COC foil in order to remove excess liquor from a crystallization drop (panels $\mathbf{A}$ and $\mathbf{C}$ ) by using a paper wick gently inserted in the same hole (panel B). Please click here to view a larger version of this figure. 


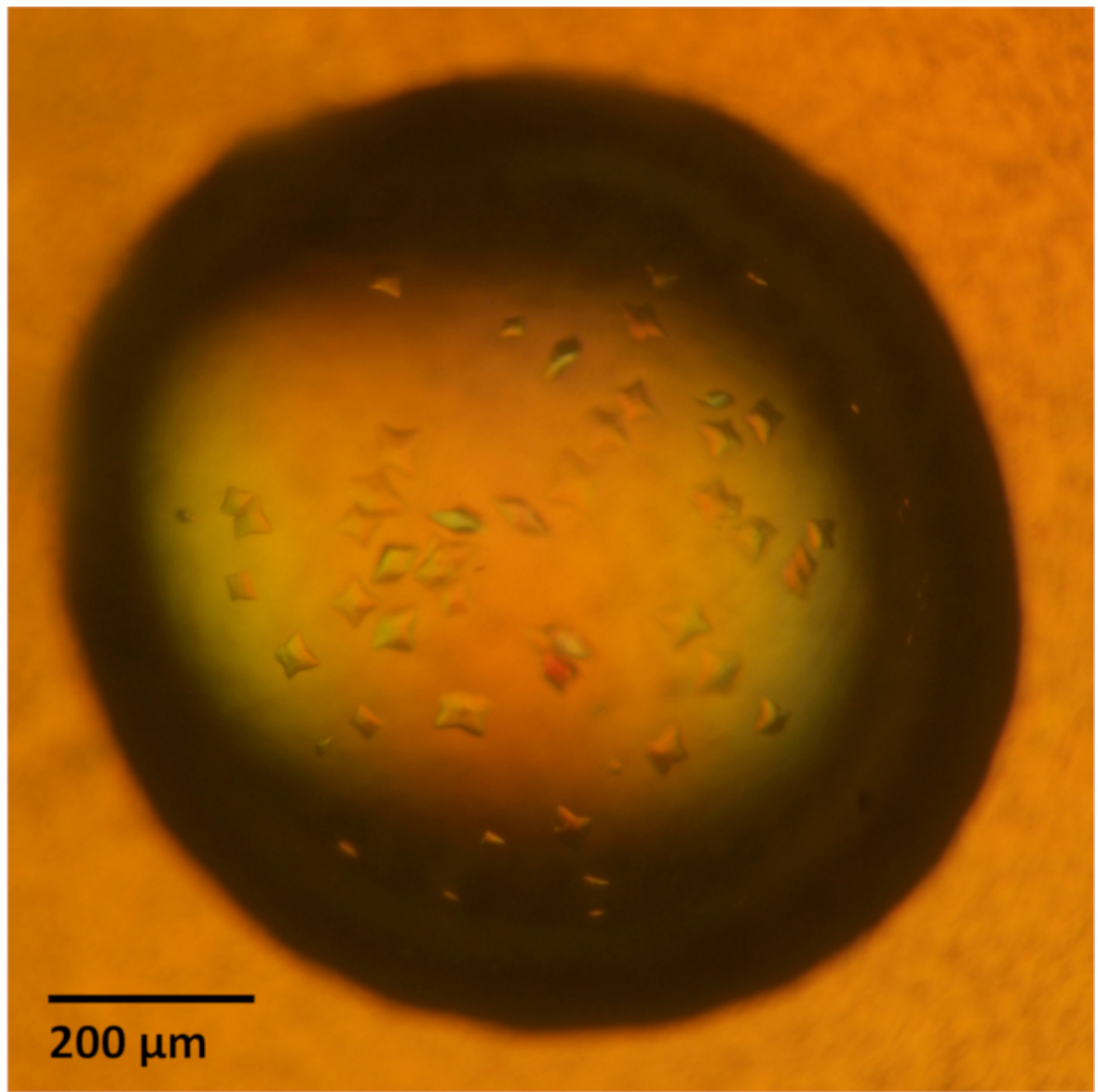

Figure 4: Image of hen egg-white lysozyme crystals observed through a transmission microscope equipped with a polarizer. Individual crystals are easily discriminated from precipitated protein solution. The crystals in this image are of an average size of $40 \mu \mathrm{m} \times 50 \mu \mathrm{m}$. Please click here to view a larger version of this figure. 


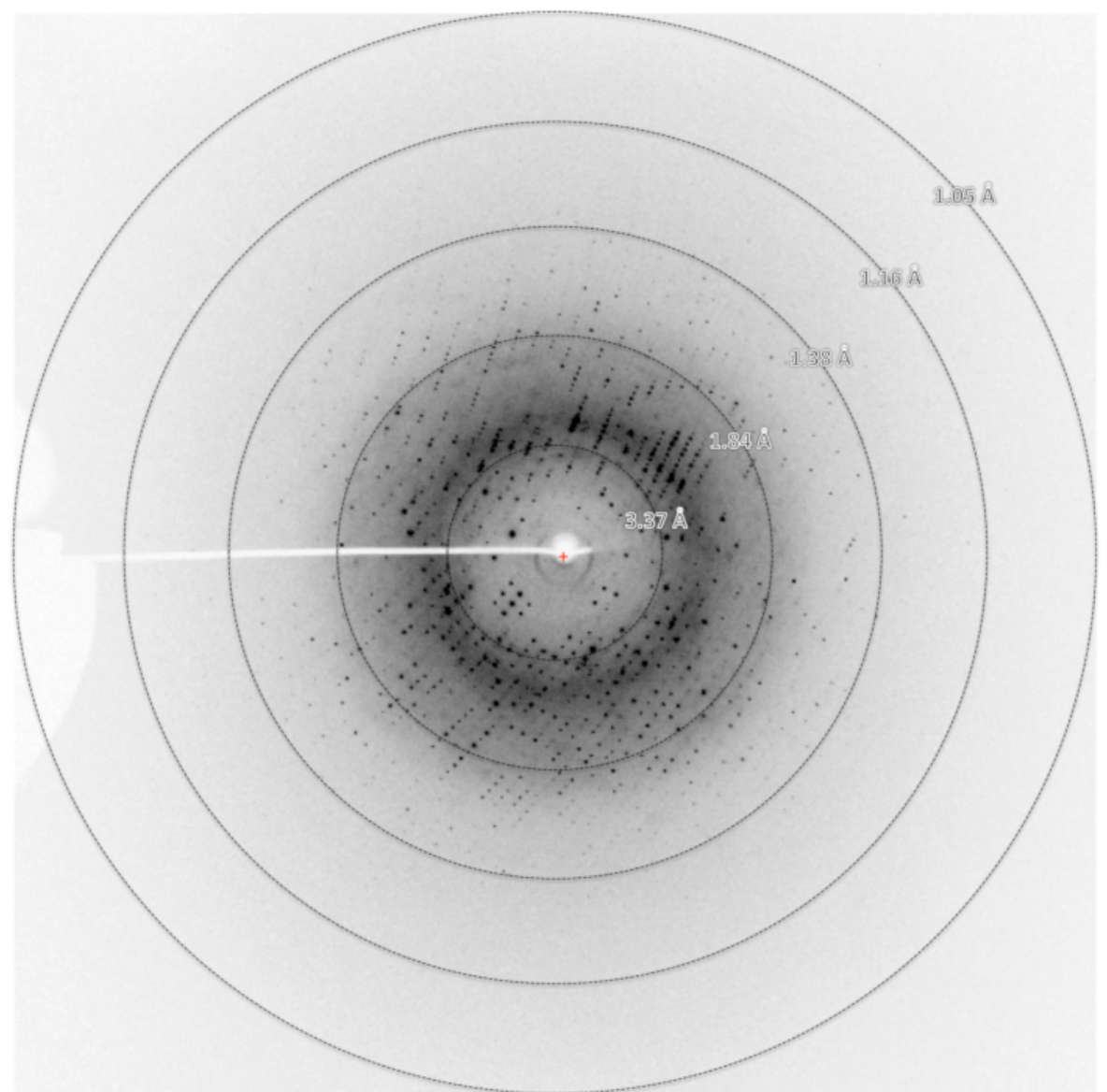

Figure 5: A typical X-ray diffraction image of a lysozyme crystal grown on the sample holder. Prior to exposure to $X$-rays all excess mother liquor was removed from around the crystal. Diffraction data were collected at ambient temperature on BL14.3 at the electron storage ring BESSY ${ }^{32}$ using a humidity controlled sample environment with $97.5 \%$ relative humidity. No elevated background due to the sample holders can be observed. The dashed lines in the image indicate the resolution rings. Please click here to view a larger version of this figure.
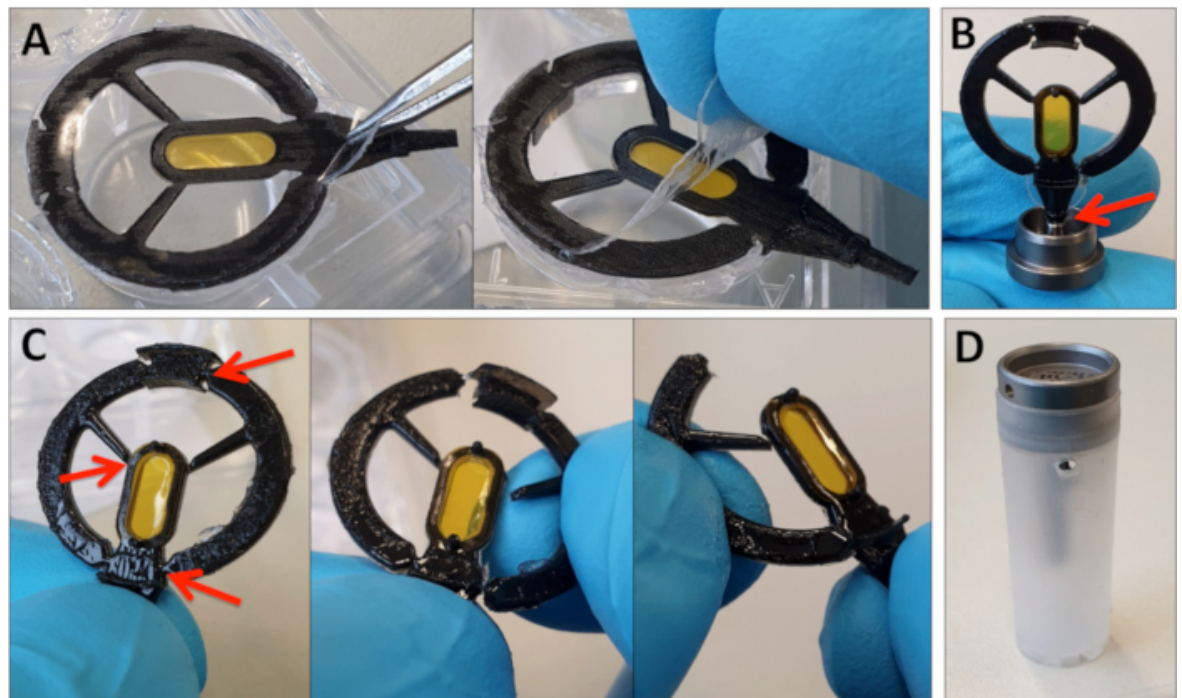

Figure 6: The sample holder is prepared for diffraction data collection. First, the COC film is lifted gently by using a forceps and then peeled off (panel A). Subsequently, the sample holder is removed from the cavity and inserted into the central hole of a magnetic base until indicated by the marker (panel B). By holding on to the central part, gentle pressure is applied to the outer ring to free the central part using the symmetrically arranged designated break points (panel C). After the removal, the sample holder can be plunged into liquid nitrogen and transferred into standard SPINE vials. Placed, for instance, in pucks they can be transported to synchrotron sites where automated sample-mounting robots recognize them as regular samples (panel $\mathbf{D}$ ). Please click here to view a larger version of this figure. 


\begin{tabular}{|l|l|}
\hline Crystallization details & \\
\hline Method & Hanging drop, vapor diffusion method \\
\hline Plate type & SuperClear Plates \\
\hline Temperature $(\mathrm{K})$ & 293 \\
\hline Protein concentration $\left(\mathrm{mg} \mathrm{mL}^{-1}\right)$ & 15 \\
\hline Composition of reservoir solution & $50 \mathrm{mM} \mathrm{NaAc} \mathrm{pH} \mathrm{4.7,500} \mathrm{mM} \mathrm{NaCl,25 \% (w/v)} \mathrm{PEG-6000}$ \\
\hline Volume and ratio of drop & $2 \mu \mathrm{L}$ total, $1: 1 \mathrm{ratio}(\mathrm{protein}: \mathrm{mother}$ liquor \\
\hline Volume of reservoir & $500 \mu \mathrm{L}$ \\
\hline Incubation time & $12 \mathrm{hours}$ \\
\hline
\end{tabular}

Table 1: Experimental details of the described crystallization experiment.

\begin{tabular}{|c|c|}
\hline Data collection and processing & \\
\hline Wavelength $(\AA)$ & 0.89429 \\
\hline Temperature (K) & 293 \\
\hline Detector & Rayonix MX225 CCD \\
\hline Crystal-detector distance (mm) & 120 \\
\hline Rotation range per image $\left(^{\circ}\right)$ & 0.5 \\
\hline Total rotation range $\left({ }^{\circ}\right)$ & 120 \\
\hline Exposure time per image (s) & 5 \\
\hline Space group & $\mathrm{P} 4{ }_{3} 2{ }_{1} 2$ \\
\hline Unit-cell parameters $(\AA)$ & $a=79.01, b=79.01, c=37.95$ \\
\hline Mosacity $\left({ }^{\circ}\right)$ & 0.07 \\
\hline Resolution range $(\AA)$ & $39.50-1.35(1.37-1.35)$ \\
\hline Total number of reflections & $191940(8932)$ \\
\hline Number of unique reflections & $27020(1292)$ \\
\hline Completeness (\%) & $99.88(99.20)$ \\
\hline Multiplicity & $7.1(6.9)$ \\
\hline Mean $\mathrm{I} / \sigma(\mathrm{I})$ & $15.0(1.9)$ \\
\hline $\mathrm{R}_{\text {meas }}{ }^{35}(\%)$ & $6.3(107.0)$ \\
\hline $\mathrm{R}_{\text {pim }}{ }^{36}(\%)$ & $2.4(40.4)$ \\
\hline $\mathrm{CC}_{1 / 2}{ }^{37}$ & $99.9(68.5)$ \\
\hline $\mathrm{ISa}^{38}$ & 16.1 \\
\hline Wilson B-factor $\left(\AA^{2}\right)$ & 17.0 \\
\hline
\end{tabular}

Table 2: Diffraction data collection and processing statistics.

\section{Discussion}

Suitability for crystallization experiments. The new sample holders can be used for standard hanging drop crystallization experiments using either 24-well Linbro type plates (types 1 and 2), or 24-well SBS footprint plates in which each well has a diameter of $18 \mathrm{~mm}$ (type 3). They can be used instead of the standard microscope cover slips. The amorphous COC foil ensures the airtightness of the system. The monitoring of the crystallization experiment is possible using a transmission light microscope, because of the use of high clarity foils. To the best of our knowledge, no other sample holders exist for 24-well crystallization plates, which would allow crystal manipulation or diffraction experiments, without mechanically removing the crystal from the drop, in which it is grown. This is of particular importance, since many researchers in the field still rely on such plates for crystal optimization, due to the fact that larger drop volumes can be used compared to 96 -well sitting-drop plates. With these larger drop volumes, larger crystals may be obtained.

Suitability for crystal manipulation. Due to the self-healing properties of the outer COC foil and the microporous structure of the inner yellow polyimide foil, the crystal environment is accessible and the crystals can be manipulated without mechanically transferring them to other containers. This makes the sample holders very convenient. The only other system we know of, which allows this indirect and gentle access to the crystal, is the CrystalDirect system ${ }^{26}$. However, CrystalDirect is less flexible since special 96-well crystallization plates have to be used. The 
foil, on which the crystals are growing, is the same that seals the crystallization experiment and it is not self-healing. This means that an aperture that has been pierced into the foil by laser ablation for ligand or cryo-protectant delivery to the crystals will remain open, increasing the chance for liquid evaporation. This is in contrast to our design, where crystals will not be directly exposed to the environment even if the COC foil gets pierced a number of times.

Suitability for in situ diffraction experiments at ambient temperature. The sample holder can be removed from the crystallization plate in a straight-forward manner, stuck onto a magnetic base and put on a beamline goniometer. For a diffraction experiment at room temperature, it is advisable to put the sample into an air stream of defined humidity ${ }^{33}$. The mother liquor around the crystal may be removed prior to putting the sample holder on the goniometer in order to reduce the background scattering. Such a set-up is stable for hours.

Suitability of the used material for operation and storage at $\mathbf{1 0 0} \mathrm{K}$. Neither the material used for the production of the sample holder nor the polyimide film are adversely affected by cooling them down to low temperatures ${ }^{34}$. Hence, working with the sample holder at low temperature (e.g., $100 \mathrm{~K}$ ) does not pose a serious problem.

Suitability for in situ diffraction experiments at $100 \mathrm{~K}$. For data collection at $100 \mathrm{~K}$ in a nitrogen stream, the sample holder needs to be removed from the crystallization plate as in the previous paragraph, stuck onto a magnetic base and put into a gaseous nitrogen stream at 100 $\mathrm{K}$ on a beamline goniometer. If desired, the sample may also be cryo-protected, although it is likely that for naked samples this may not be necessary in most cases ${ }^{31}$. For experiments at $100 \mathrm{~K}$, the sample holders type 2 and 3 are better suited because the outer plastic ring can be removed. Hence, they are of smaller size and should therefore be less prone to icing. However, even a sample holder of type 1 may be used. Given a not too high humidity in the experimental hutch and a properly aligned cryo-system icing up of the holder is not really a problem.

Limitations. The sample holder's geometry permits unobstructed diffraction data collection by the rotation method over a total rotation range of $160^{\circ}$. This is sufficient so that complete diffraction data sets can be obtained for most crystal systems. In cases where this is not possible, data from more than crystal need to be merged together. When crystals are grown together, it may be possible to adjust the size of the incident X-ray beam so that only parts of individual crystals are exposed. In extreme cases, one may need to resort to a data collection strategy similar to the MeshAndCollect approach ${ }^{35}$. In summary, while there are certain limitations associated with the sample holders, these can be overcome in most cases. Of course, it is always possible that situations are encountered, in which none of this is possible. In such cases, one may need to resort to other crystal mounting methods.

We have described a novel type of sample holder for macromolecular crystallography and we have demonstrated the suitability of the sample holders for various applications. Taking into account the simple and reproducible handling of protein crystals, as well as the unique properties of the sample holders, we believe that these sample holders will prove to be a valuable addition to the arsenal of sample holders for macromolecular crystallography.

\section{Disclosures}

Patent applications regarding the reported sample holder have been filed by Helmholtz-Zentrum Berlin with the following registration numbers and registration dates with the German Patent and Trademark Office: DE 102018129 125.6, registration date November $20^{\text {th }}$, 2018 ; DE 10 2018125 129.7, registration date October 11 ${ }^{\text {th }}$, 2018; DE 102017129761.8 , registration date December $13^{\text {th }}$, 2017. A subsequent international patent application via the PCT route, using the priority of DE 102017129761.8 has been filed, PCT/DE2018/101007. A registration of a utility model with the number DE 202018106955.1 was filed on December $6^{\text {th }}, 2018$. The sample holder has been made commercially available under the trade names XtalTool and XtalTool/HT by Jena Bioscience, Jena, Germany.

\section{Acknowledgments}

The authors would like to thank BESSY II, operated by Helmholtz-Zentrum Berlin for beam time access and support, and the departments of Sample Environment and Technical Design for their help with design and construction and the access to the 3D-printer facilities.

\section{References}

1. Berman, H.M. et al. The Protein Data Bank. Nucleic Acids Research. 28 (1), 235-242 (2000)

2. Mac Sweeney, A., D'Arcy, A. A simple and rapid method for mounting protein crystals at room temperature. Journal of Applied Crystallography. 36 (1), 165-166 (2003).

3. Kalinin, Y. et al. A new sample mounting technique for room-temperature macromolecular crystallography. Journal of Applied Crystallography. 38 (2), 333-339 (2005).

4. Basavappa, R., Petri, E.T., Tolbert, B.S. A quick and gentle method for mounting crystals in capillaries. Journal of Applied Crystallography. 36 (5), 1297-1298 (2003).

5. Pflugrath, J.W. Macromolecular cryocrystallography-methods for cooling and mounting protein crystals at cryogenic temperatures. Methods. 34 (3), 415-423 (2004).

6. Garman, E.F., Schneider, T.R. Macromolecular Cryocrystallography. Journal of Applied Crystallography. 30 (3), $211-237$ (1997).

7. Gavira, J.A., Toh, D., Lopéz-Jaramillo, J., García-Ruiz, J.M., Ng, J.D. Ab initio crystallographic structure determination of insulin from protein to electron density without crystal handling. Acta Crystallographica Section D: Biological Crystallography. 58 (7), 1147-1154 (2002).

8. Martínez-Rodríguez, S. et al. Crystallization and preliminary crystallographic studies of an active-site mutant hydantoin racemase from Sinorhizobium meliloti CECT4114. Acta Crystallographica Section F: Structural Biology and Crystallization Communications. 64 (Pt 1), $50-53$ (2007).

9. Hope, H. Cryocrystallography of biological macromolecules: a generally applicable method. Acta Crystallographica Section B: Structural Science. 44 (1), 22-26 (1988). 
10. Teng, T.-Y. Mounting of crystals for macromolecular crystallography in a free-standing thin film. Journal of Applied Crystallography. 23 (5), 387-391 (1990).

11. Thorne, R.E., Stum, Z., Kmetko, J., O'Neill, K., Gillilan, R. Microfabricated mounts for high-throughput macromolecular cryocrystallography. Journal of Applied Crystallography. 36 (6), 1455-1460 (2003).

12. Jian-Xun, Q., Fan, J. An improved loopless mounting method for cryocrystallography. Chinese Physics B. 19 (1), 010601 (2010).

13. Kitatani, T. et al. New Technique of Manipulating a Protein Crystal Using Adhesive Material. Applied Physics Express. 1 (3), 037002 (2008).

14. Mazzorana, M., Sanchez-Weatherby, J., Sandy, J., Lobley, C.M.C., Sorensen, T. An evaluation of adhesive sample holders for advanced crystallographic experiments. Acta Crystallographica Section D: Biological Crystallography. 70 (Pt 9), 2390-2400 (2014).

15. Wierman, J.L., Alden, J.S., Kim, C.U., McEuen, P.L., Gruner, S.M. Graphene as a protein crystal mounting material to reduce background scatter. Journal of Applied Crystallography. 46 (5), 1501-1507 (2013).

16. Parkin, S., Hope, H. Macromolecular Cryocrystallography: Cooling, Mounting, Storage and Transportation of Crystals. Journal of Applied Crystallography. 31 (6), 945-953 (1998).

17. Papp, G. et al. Towards a compact and precise sample holder for macromolecular crystallography. Acta Crystallographica Section D: Structural Biology. 73 (10), 829-840 (2017).

18. Roedig, P. et al. A micro-patterned silicon chip as sample holder for macromolecular crystallography experiments with minimal background scattering. Scientific Reports. 5, 10451 (2015).

19. Roedig, P. et al. Room-temperature macromolecular crystallography using a micro-patterned silicon chip with minimal background scattering. Journal of Applied Crystallography. 49 (3), 968-975 (2016)

20. Zarrine-Afsar, A. et al. Crystallography on a chip. Acta Crystallographica Section D: Biological Crystallography. 68 (3), $321-323$ (2012).

21. Mueller, C. et al. Fixed target matrix for femtosecond time-resolved and in situ serial micro-crystallography. Structural Dynamics. 2 (5), 054302 (2015).

22. Feld, G.K. et al. Low-Z polymer sample supports for fixed-target serial femtosecond X-ray crystallography. Journal of Applied Crystallography. 48 (4), 1072-1079 (2015).

23. le Maire, A. et al. In-plate protein crystallization, in situ ligand soaking and X-ray diffraction. Acta Crystallographica Section D: Biological Crystallography. 67 (9), 747-755 (2011).

24. Soliman, A.S.M., Warkentin, M., Apker, B., Thorne, R.E. Development of high-performance X-ray transparent crystallization plates for in situ protein crystal screening and analysis. Acta Crystallographica Section D: Biological Crystallography. 67 (7), $646-656$ (2011).

25. Aller, P. et al. Application of in situ diffraction in high-throughput structure determination platforms. Methods in Molecular Biology (Clifton, N.J.). 1261, 233-253 (2015).

26. Cipriani, F., Röwer, M., Landret, C., Zander, U., Felisaz, F., Márquez, J.A. CrystalDirect: a new method for automated crystal harvesting based on laser-induced photoablation of thin films. Acta Crystallographica. Section D, Biological Crystallography. 68 (Pt 10), 1393-1399 (2012).

27. Zander, U. et al. Automated harvesting and processing of protein crystals through laser photoablation. Acta Crystallographica Section D: Structural Biology. 72 (4), 454-466 (2016)

28. Antimonov, M. et al. Large-area Kapton x-ray windows. Advances in X-Ray/EUV Optics and Components X. 9588, 95880F (2015).

29. McPherson, A. Penetration of dyes into protein crystals. Acta Crystallographica Section F: Structural Biology Communications. 75 (2), 132-140 (2019).

30. Bowler, M.G., Bowler, D.R., Bowler, M.W. Raoult's law revisited: accurately predicting equilibrium relative humidity points for humidity control experiments. Journal of Applied Crystallography. 50 (2), 631-638 (2017).

31. Pellegrini, E., Piano, D., Bowler, M.W. Direct cryocooling of naked crystals: are cryoprotection agents always necessary? Acta Crystallographica Section D: Biological Crystallography. 67 (10), 902-906 (2011).

32. Mueller, U. et al. The macromolecular crystallography beamlines at BESSY II of the Helmholtz-Zentrum Berlin: Current status and perspectives. The European Physical Journal Plus. 130 (7), 141 (2015).

33. Bowler, M.W. et al. Automation and Experience of Controlled Crystal Dehydration: Results from the European Synchrotron HC1 Collaboration. Crystal Growth \& Design. 15 (3), 1043-1054 (2015).

34. Yano, O., Yamaoka, H. Cryogenic properties of polymers. Progress in Polymer Science. 20 (4), 585-613 (1995).

35. Zander, U. et al. MeshAndCollect: an automated multi-crystal data-collection workflow for synchrotron macromolecular crystallography beamlines. Acta Crystallographica. Section D, Biological Crystallography. 71 (Pt 11), 2328-2343 (2015). 